\title{
Migration et (contre)révolution dans le Golfe : politiques migratoires et politiques de l'emploi en Arabie saoudite
}

Migration and (Counter)Revolution the Arab Gulf: Migration Policies and Labour Market Policies in Saudi Arabia

Migración y (contra)revolución en el Golfo: políticas migratorias y políticas de empleo en Arabia Saudita

\section{Hélène Thiollet}

\section{(2) OpenEdition \\ Journals}

Édition électronique

URL : https://journals.openedition.org/remi/7400

DOI : $10.4000 /$ remi.7400

ISSN : $1777-5418$

Éditeur

Université de Poitiers

\section{Édition imprimée}

Date de publication : 1 décembre 2015

Pagination : 121-143

ISBN : 979-10-90426-26-9

ISSN : 0765-0752

\section{Référence électronique}

Hélène Thiollet, « Migration et (contre)révolution dans le Golfe : politiques migratoires et politiques de l'emploi en Arabie saoudite », Revue européenne des migrations internationales [En ligne], vol. 31 - n³ et 4 | 2015, mis en ligne le 01 décembre 2018, consulté le 14 avril 2022. URL : http:// journals.openedition.org/remi/7400 ; DOI : https://doi.org/10.4000/remi.7400 


\section{Migration et (contre)révolution dans le Golfe : politiques migratoires et politiques de l'emploi en Arabie saoudite}

\section{Hélène Thiollet ${ }^{1}$}

Tilly (1973) remarquait qu'expliquer une révolution a souvent pour objectif soit de prédire, soit d'empêcher les prochaines...; ;'analyse des causes d'une révolution devient alors une préoccupation de météorologue ou d'ingénieur. Alors que des mouvements sociaux mettaient à mal les autoritarismes du Moyen-Orient, les monarchies du Golfe et en particulier I'Arabie saoudite, ont déployé à l'échelle nationale et internationale une ingénierie sociale qui s'attaque aux causes structurelles supposées des printemps arabes pour empêcher leur éclosion dans le Golfe. À partir de 2011, l'Arabie saoudite se lance en effet dans une vague intense de répression des opposants au régime et développe un arsenal de politiques sociales visant à réduire les facteurs structurels de mécontentement : le chômage essentiellement, les problèmes de logement en milieu urbain et les prix, via une augmentation des salaires dans le secteur public et privé. Plusieurs questions apparaissent ou réapparaissent en 2011 comme centrales dans le dispositif de politiques publiques " contre-révolutionnaires ": la dépendance structurelle des économies rentières à la maind'œuvre immigrée, la compétition entre travailleurs expatriés et main-d'œuvre locale et le risque que représentent les populations étrangères qui dominent la démographie des marchés du travail, voire supplantent largement les effectifs des populations nationales.

Les pays du Golfe, et en particulier l'Arabie saoudite, constituent un cas d'étude souvent négligé des " printemps arabes " et cela pour deux raisons. Tout d'abord, la plupart des observateurs considèrent qu'il n'y a pas eu de printemps arabe dans le Golfe et pas de " printemps saoudien " dans le royaume (De Bel-Air, 2012 : 87). Pourtant, des soulèvements ont bien eu lieu, même si la répression menée en Arabie saoudite, au Bahreïn, avec l'aide de I'Arabie saoudite, ou en Oman a brutalement et efficacement réprimé les dyna-

1 Chargée de recherche CNRS (Research fellow CNRS), 56 rue Jacob, 75006 Paris ; helene.thiollet@sciencespo.fr

Je remercie Nicholas van Hear et Oliver Bakewell pour leurs commentaires à la suite d'une intervention au séminaire COMPAS "Shifting powers, shifting mobilities " (Oxford, 5 mars 2015). Je remercie aussi chaleureusement Delphine Pagès-El Karoui co-éditrice de ce dossier thématique pour ses relectures et ses commentaires. 
miques contestataires. L'invisibilité politique de ces mouvements d'opposition tient peut-être à leur dimension confessionnelle - comme en Arabie ou à Bahreïn (Coates Ulriksen, 2013). Pourtant, ces mouvements avaient aussi une dimension politique (Lacroix, 2014) et souvent une base de revendications salariales et socio-économiques au sens large.

La deuxième raison est que des observateurs, distants de sociétés considérées comme " riches", minimisent souvent les facteurs économiques et sociaux de mécontentement et les revendications suscitées par la crise économique documentées notamment par les enquêtes de l'Arab Barometer, l'augmentation des prix notamment des denrées alimentaires, le chômage des jeunes diplômés (Shafeeq et al., 2014), le " youth bulge " ou explosion de la classe d'âge vingtquarante ans, liée à la fin de la transition démographique (Hoffman et Amaney, 2012).

Dans cet article, il s'agit d'abord de revenir sur le lien entre migrations et révolutions, à travers ses manifestations dans le Golfe. On s'intéressera particulièrement à la façon dont les États du Golfe ont envisagé les immigrés comme facteur de perturbation politique dès les années 1950. II ressort de cette analyse que c'est moins l'acception de révolution entendue comme " changement de régime " que l'on retient comme pertinente pour comprendre le lien entre migration et révolution dans le Golfe (Thiollet, 2013). La notion de " révolution " mobilisée dans cet article est celle de " révolution sociale ", entendue comme " transformation rapide et basique des structures de l'État et des classes sociales, accompagnée et en partie menée par des révoltes de classe "par le bas" " (Skocpol, $1979:$ 287).

Puis on analysera les politiques migratoires et les politiques sociales déployées au cours des printemps arabes dans les pays du Golfe, à travers le cas de l'Arabie saoudite. Sur la base de sources administratives et d'une enquête qualitative menée dans ce pays ${ }^{2}$, on avance que les politiques migratoires déployées dans le Golfe depuis les printemps arabes constituent une forme de prophylaxie contre-révolutionnaire. Pour en saisir le sens, les politiques de 2011 sont replacées dans une tendance longue des politiques migratoires golfiennes depuis le début des années 1990. La migration est construite dans le champ politique comme une menace existentielle pour les régimes fragilisés, en particulier en Arabie saoudite où le chômage des jeunes diplômés s'installe comme une donnée durable du marché du travail. À grand renfort de subventions pour l'emploi des citoyens et d'expulsions des travailleurs étrangers et de leurs familles, l'Arabie saoudite tente de modeler la société et le marché du travail. On montre néanmoins que les politiques golfiennes et saoudienne en particulier, sont moins des politiques " anti-immigration " que des politiques " anti-immigrés ", fondées sur le contrôle et la fragilisation de sa très nombreuse population étrangère.

2 L'enquête qualitative, menée avec le soutien des programmes de recherche SYSREMO et MOBGLOB financés par l'Agence Nationale pour la Recherche et le Faisal Center for Research and Islamic Studies de Riyad, compte une cinquantaine d'entretiens menés entre 2013 et 2015 auprès de ministères, d'agences publiques, d'entrepreneurs du secteur privé et de chambres de commerce en Arabie saoudite. 


\section{Migration et révolution}

\section{Les printemps du Golfe : de la répression à la " contre-révolution "}

De nombreux analystes se sont intéressés aux interventions extérieures, aux aspects diplomatiques et purement répressifs de la politique saoudienne face aux printemps arabes depuis 2011 en s'interrogeant sur son caractère " contrerévolutionnaire " (Gause, 2011 ; Kamrava, 2012 ; Steinberg, 2014). L'Arabie saoudite mène dès 2011 une politique d'intervention sur la scène régionale, au Bahreïn, en Tunisie, en Égypte et au Yémen. L'intervention militaire saoudienne pour réprimer les manifestations pro-démocratie et contre la dynastie des Al Khalifa du 23 février à Bahreïn en est la forme la plus aiguë. II existe un lien fort entre la dimension interne - répression et musellement par anticipation d'un mouvement de contestation du pouvoir - et internationale - stratégie de défense des intérêts saoudiens contre I'Iran dans une confrontation formulée comme un antagonisme religieux opposant chiites et sunnites. Dans cet article, on utilise le terme " contre-révolution " pour qualifier deux types de politiques saoudiennes : les politiques de répression des acteurs sociaux (agency) et celles qui tentent d'identifier, de contrôler et de transformer les facteurs d'émergence de mouvements sociaux contestataires (structures). On s'intéressera particulièrement dans cet article aux secondes. L'analyse de la séquence de politiques publiques de 2011-2015 en réponse aux printemps arabes implique néanmoins de revenir sur l'analyse du lien plus théorique établi par les acteurs entre " migrations et révolutions".

Le " printemps saoudien " a revêtu trois caractéristiques spécifiques : - II a été structuré par des dynamiques religieuses, essentiellement par l'opposition islamiste réformiste au régime saoudien (al-Sahwa al-Islamiya, "le réveil musulman " ou mouvement salafiste réformiste lié aux Frères musulmans) - des sunnites donc $-{ }^{3}$ et par une population jeune et chiite de l'Est du pays réclamant l'égalité en matière de droits sociaux, économiques et politiques avec les sunnites. La tradition d'opposition aux discriminations dont sont victimes les chiites en Arabie (Matthiessen, 2013) est à la fois religieuse, politique et géopolitique et s'était cristallisée en un mouvement social dès 1979, à la suite de la révolution islamique.

- Il a été brutalement réprimé " par anticipation ", dès 2011, tant pour les islamistes sunnites et les libéraux ${ }^{4}$ que pour les populations chiites.

- Il a donné lieu à une politique de cooptation et de distribution de prébendes à la fois ciblée et collective, momentanée et structurelle, qui l'a empêché de se déployer en étouffant les foyers de contestation dans l'ensemble du pays, à I'exception d'une poche dans I’Est chiite. Ce dispositif avait déjà permis à

\footnotetext{
3 Le soutien précoce des islamistes saoudiens aux mouvements révolutionnaires en Égypte et en Tunisie est ancré dans la solidarité idéologique de la Sahwa avec les organisations locales liées aux Frères musulmans depuis les années 1970. Ceux-ci sont par ailleurs restés en majorité hostiles aux mouvements de contestations initiés par les chiites dans l’Est du pays, accusés de rébellion " sectaire " génératrice de chaos (fitna) et non d'œuvre pour une révolution nationale.

4 Le " jour de rage " prévu en février 2011 à l'instar des jours de manifestations égyptiens et tunisiens a été " tué dans l'œuf " par une répression brutale des opposants islamistes comme libéraux dans l'ensemble du pays et n'a pu " éclore " que dans l'Est du pays.
} 
la famille régnante d'apaiser une partie du mouvement d'opposition, né de la première guerre en Irak et de la présence des troupes américaines sur le sol saoudien en 1991.

\section{De la politisation des immigrés à la politisation de l'immigration}

Le Moyen-Orient est une zone de forte circulation, mais de faible intégration formelle des migrants dans les sociétés et systèmes politiques des pays d'accueil. La région est historiquement caractérisée à la fois par une très forte circulation migratoire intra-régionale entre les années 1950 et 1980 (Beaugé et Roussillon, 1988 ; Shafik, 1999 ; Fargues, 2000) et par un faible degré d'encadrement juridique et politique de cette mobilité (Thiollet, 2011). Le contrôle des communautés étrangères a été rapidement mis au centre des politiques de " gestion " des migrants : les immigrés politisés sont considérés comme les cinquièmes colonnes de puissances étrangères, des " agitateurs " étrangers ou " ennemis de l'intérieur ". La dimension exogène des mouvements sociaux de contestation est souvent mise en avant par les pouvoirs coloniaux puis par les monarchies du Golfe pour délégitimer leur opposition : le thème des " foreign agitators " est récurrent dans les archives de compagnies pétrolières comme I'ARAMCO en Arabie saoudite (Seccombe et Lawless, 1986). Vitalis (2007) a mis en cause la propagande menée par I'ARAMCO à propos des grèves de 1953 et 1956 dans l'industrie pétrolière saoudienne en rappelant que les acteurs principaux des grèves sont saoudiens. II n'en reste pas moins que la diffusion révolutionnaire des révolutions nationalistes arabes (égyptienne, irakienne et syrienne), notamment via la diffusion des idéologies panarabes par les intellectuels migrants a poussé les monarchies du Golfe à une répression sanglante à l'époque, à l'instar de ce que l'on voit aujourd'hui dans le cadre des printemps arabes (Weyland, 2012). Comme I'ont montré Chalcraft (2011) et Brand (1988), les migrants arabes arrivés en masse à partir des années 1950 ont contribué à la diffusion du nationalisme arabe et d'idéologie marxistes dans la péninsule arabique, notamment via l'activisme syndical ou leur présence dans les métiers de l'enseignement. Matthiesen (2014 : 477) a aussi documenté la " contagion idéologique " et la diffusion du panarabisme en Arabie saoudite entre 1950 et 1975. Elle s'opère grâce aux migrations de travail et aux liens familiaux entre chiites de I'Est de l'Arabie, de Bahreïn et d'Irak, illustrant non pas I'activisme des migrants, mais les " transferts sociaux et culturels " (social remittances), $c^{\prime}$ est-à-dire à la circulation de " structures normatives, de systèmes de pratiques, d'identités, et de capital social " (Levitt, 1998 : 933).

Les monarchies du Golfe ont donc, dès les années 1960, conjugué la répression momentanée des grèves et des mouvements politiques souterrains de l'opposition de gauche panarabe avec l'institutionnalisation d'une politique de séparation des étrangers et des citoyens. Ces politiques ont été soutenues, voire instiguées, par les grandes multinationales pétrolières américaines et leurs équivalents anglais dans les monarchies du Golfe sous mandat britannique jusqu'en 1971. Vitalis y voit l'importation du système de ségrégation Jim Crow dans le contexte golfien. Elles imposent un mode de sociabilité ségrégé qui préside aujourd'hui encore aux rapports sociaux entre migrants et nationaux (Thiollet, 2010b) et garantit l'exploitation des premiers par les seconds, doublement " rentiers ", du pétrole et du travail des étrangers (Ibrahim, 1982). Dans un 
contexte d'immigration massive et de circulation des populations, le contrôle des " ennemis de l'intérieur " s'exerce à travers ces structures de hiérarchisation et de ségrégation socio-spatiale et à travers la répression de toute forme d'activisme politique ou syndical. Les pratiques de contrôle et d'exclusion dans des États où l'intégration juridique et politique, et I'assimilation culturelle et identitaire est considérée comme difficile, voire impossible, participent d'une construction globale des rapports entre État et société où les migrants sont invisibles, exclus, mais essentiels comme le formule Okruhlik (1999). Produit de cette histoire, le " modèle golfien " en matière migratoire est aujourd'hui axé sur des politiques " anti-intégration ", dont l'objet est d'empêcher l'intégration des travailleurs migrants dans les sociétés d'accueil au nom d'une définition étroite de la nation (Longva, 2005 ; Thiollet, 2010b). La convergence des politiques migratoires des pays du Golfe est renforcée par coordination des politiques migratoires au sein du Conseil de coopération du Golfe (CCG) (Winckler, 1997).

Contrairement aux années 1950, où la contestation de l'exploitation rejoignait des mobilisations politiques contre les régimes monarchiques et empruntait au répertoire de l'idéologie socialiste panarabe, la période contemporaine est caractérisée par des revendications presque inaudibles et exclusivement économiques. L'“ activisme " des immigrants des pays du Golfe est récent, observé surtout à Dubaï en 2005, en 2007 avec une grève de près de 30000 travailleurs étrangers contre l'entreprise de construction Arabtec, ou encore en 2008. Ces mouvements de travailleurs étrangers sont largement ignorés des médias et des chercheurs. Leurs protestations contre les abus dont ils sont victimes (confiscation des passeports, conditions de travail dangereuses, conditions d'hébergement insalubres, etc.) et pour la défense de leurs droits économiques et sociaux (paiement des salaires, augmentations) tranchent avec les " soulèvements visant à réformer l'État et obtenir une reconnaissance de droits politiques " (Kanna, 2012 : 147).

Ces mobilisations socio-économiques échappent a priori à la catégorie de "révolution " entendue au sens simple de changement de régime. En revanche, la notion large ou maximaliste de "révolution sociale " s'impose pour comprendre le "risque " couru par les sociétés golfiennes face au phénomène migratoire. Un risque " existentiel " si l'on se réfère aux questions d'identité et au risque du multiculturalisme, ou un risque que l'on peut qualifier de structurel. En ce sens, lorsque Skocpol envisage les révolutions française, russe et chinoise comme la " transformation rapide et basique des structures de l'État et des classes sociales, accompagnée et en partie menée par des révoltes de classe "par le bas" "(Skocpol, $1979: 287$ ), elle dessine un avenir possible de transformation des rapports de classe dans le Golfe. La segmentation entre étrangers et nationaux/citoyens, mais aussi des différenciations à l'intérieur de ces deux groupes sont considérées comme structurant les relations sociales, économiques et politiques au sein de la société saoudienne. La préoccupation de l'État vis-à-vis des " agitateurs étrangers " dans les mobilisations sociales des années 1950 ou des printemps arabes reste importante, mais la " politisation des migrations " change de sens entre les années 1970 et la période contemporaine. L'enjeu central des politiques publiques n'est plus exclusivement de contrôler les mobilisations des étrangers, mais aussi et surtout de contrôler leur poids économique et social, leur "intégration " de fait sinon en droit dans les pays du Golfe. La politisation des immigrés cède le pas à la politisation de l'immigration comme phénomène structurel. 
En s'intéressant aux modalités de politisation de la question migratoire en Arabie saoudite au cours de I'histoire récente, on redonne une profondeur socio-historique à l'examen du lien entre migrations et révolution. Pour ce faire, on propose dans les parties suivantes d'articuler crises politiques (1991, et l'invasion du Koweït ; 2011, et les printemps arabes) et tendances longues ou institutionnelles de la politisation des migrations.

\section{La gestion des migrations en Arabie saoudite : généalogie d'une ingénierie sociale}

\section{Le modèle golfien : politiques anti-immigration et déni d'immigration}

Les pays exportateurs de pétrole rassemblés au sein du CCG ont connu un développement économique rapide, fondé sur l'importation massive de main$d$ 'œuvre étrangère qualifiée et non qualifiée, majoritairement venue d'autres pays du "Sud ". Le CCG a les ratios de population immigrée les plus importants de la planète, entre un tiers et $89 \%$ des résidents selon les pays (voirTableau 1). La région du Golfe compte plus de $60 \%$ de travailleurs étrangers dans sa population active. Les migrants venaient en majorité du monde arabe et des pays développés entre les années 1950 et 1980, et d'Asie à partir des années 1980. Les taux d'immigration nette baissent fortement au tournant des années 1980, mais ils sont positifs et stables entre trois et dix pour mille depuis, malgré la crise de 1991 (voir Graphique 1). Le flux continu de main-d'œuvre et l'accroissement naturel assurent une croissance continue de la population étrangère en Arabie (Graphique 2).

En dépit de la manipulation flagrante des données statistiques fournies par les pays de la région et de l'inexistence de données fiables produites à l'extérieur, ces ordres de grandeur indiquent assez l'ampleur du facteur migratoire comme déterminant des équilibres sociaux et démographiques dans les pays du CCG. L'Arabie saoudite fait figure de géant démographique et géographique dans la région et se distingue par sa prééminence en matière de production pétrolière à l'échelle mondiale ; son poids politique au Moyen-Orient et la part de l'immigration arabe (yéménite, égyptienne, jordano-palestinienne) y sont plus importants que dans d'autres monarchies du Golfe, majoritairement peuplées de communautés asiatiques.

La "gestion " de l'immigration dans les pays du CCG se focalise sur le contrôle des " stocks " plutôt que sur la limitation des "flux ". Elle vise à garantir la distinction de deux catégories de la population, les étrangers et les nationaux, dans un contexte d'immigration de travail massive toujours présentée et traitée comme temporaire. Le modèle migratoire golfien tel qu'il s'est consolidé au début des années 1990, repose sur la limitation de la durée des séjours et du regroupement familial, la quasi-impossibilité d'accéder aux droits politiques et à la citoyenneté par la voie de la naturalisation, la limitation des droits économiques et sociaux des migrants, la ségrégation spatiale et sociale des immigrés d'avec les nationaux et l'expulsion massive des travailleurs immigrés illégaux. 
Graphique 1 : Taux d'immigration nette 1960-2010 (pour 1000 habitants)

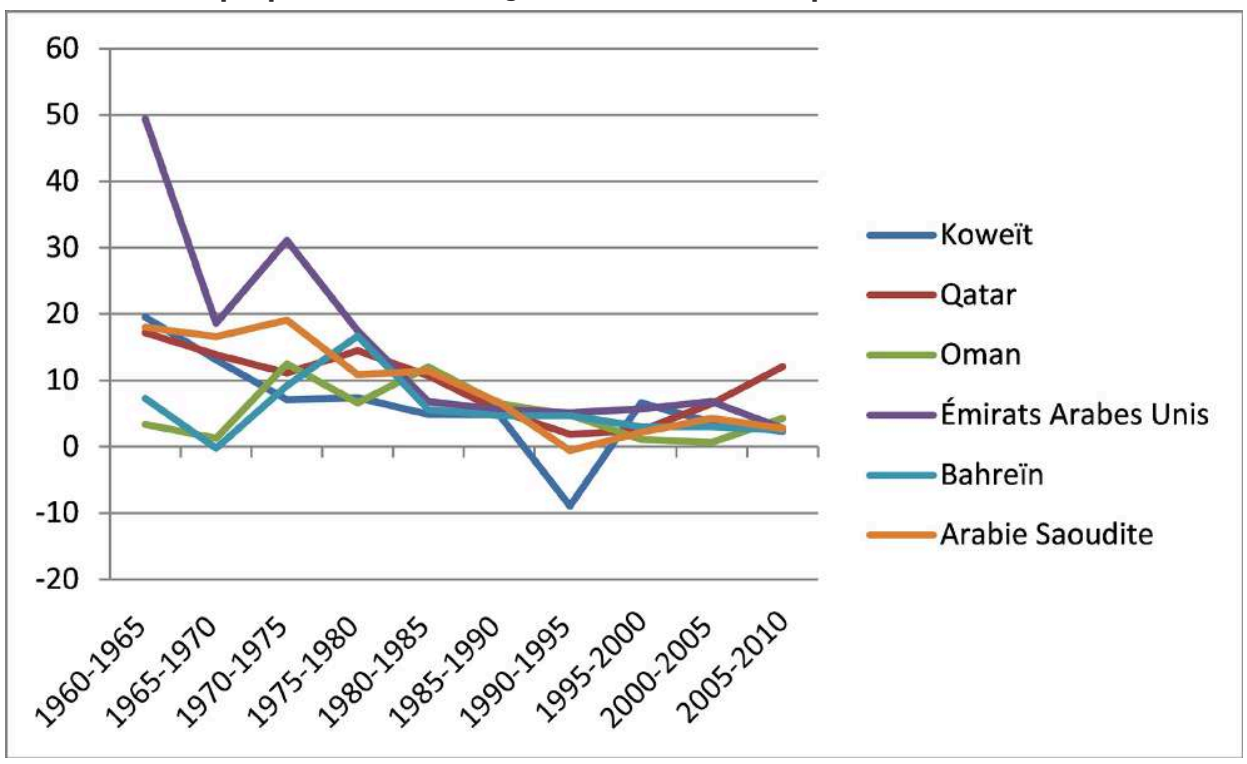

Source : United Nations, Department of Economic and Social Affairs, Population Division, basé sur Trends in International Migrant Stock.

Graphique 2 : Part des migrants dans la population saoudienne (1960-2010)

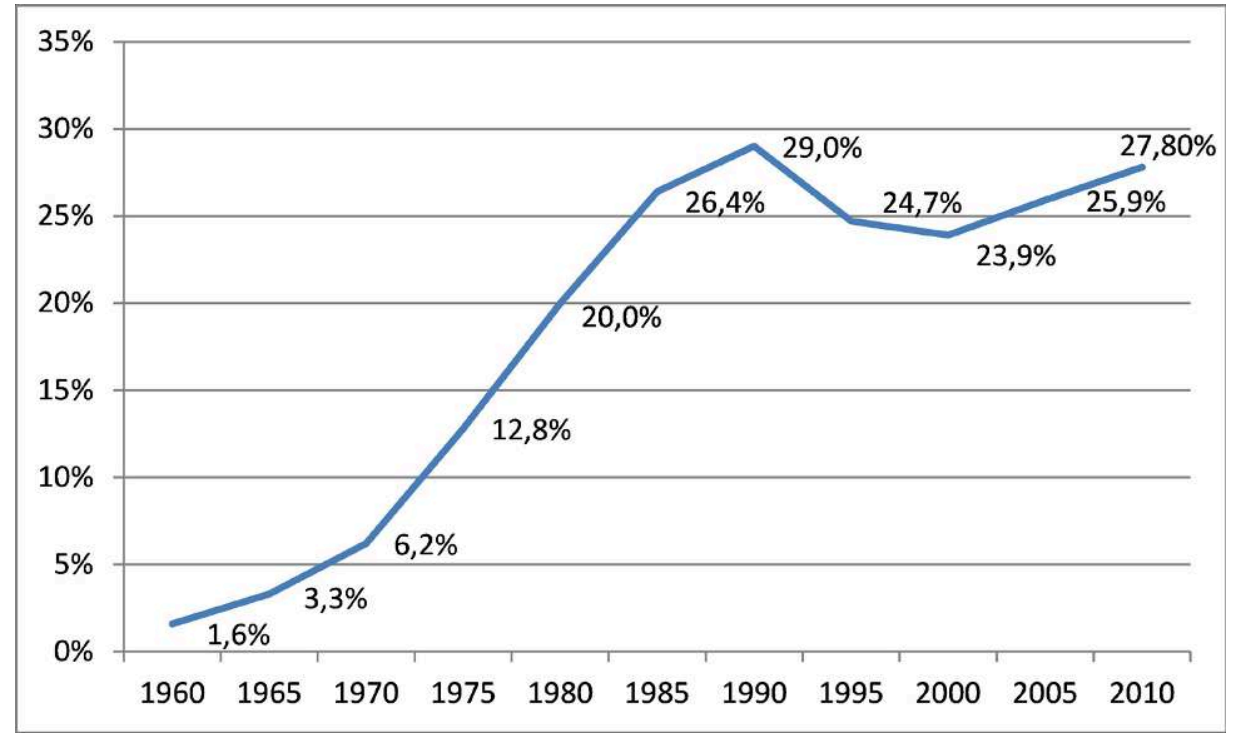

Source : United Nations, Department of Economic and Social Affairs, Population Division, basé sur Trends in International Migrant Stock. 
Tableau 1 : Populations des pays du Conseil de Coopération du Golfe, étrangers et citoyens en fonction des derniers recensements

\begin{tabular}{|c|c|c|c|c|c|}
\hline & Citoyens & $\%$ & Étrangers & $\%$ & $\begin{array}{l}\text { Population } \\
\text { totale }\end{array}$ \\
\hline $\begin{array}{l}\text { Bahreïn (census 2011, Central Information } \\
\text { Office/CIO) }\end{array}$ & 584688 & 49 & 610332 & 51 & 1195020 \\
\hline $\begin{array}{l}\text { Qatar (données disponibles QSA pour la } \\
\text { population totale, recensement de } 2010 \\
\text { et ESCWA-UN report on World Migration } \\
\text { Stocks pour les migrants) }\end{array}$ & 243267 & 14 & 1456168 & 86 & 1699435 \\
\hline Oman $(2013$, mid year estimates NCSI) & 2172002 & 56 & 1683204 & 44 & 3855206 \\
\hline $\begin{array}{l}\text { Koweït (2011, Public Authority for Civil } \\
\text { Information, PACI) }\end{array}$ & 1258254 & 31 & 2781191 & 69 & 4039445 \\
\hline $\begin{array}{l}\text { Émirats Arabes Unis (2010, National } \\
\text { Bureau of Statistics) }\end{array}$ & 947997 & 11 & 7316073 & 89 & 8264070 \\
\hline $\begin{array}{l}\text { Arabie saoudite (2013, SAMA } \\
\text { estimations) }\end{array}$ & 20271058 & 68 & 9723214 & 32 & 29994272 \\
\hline Total CCG & 25477266 & 52 & 23570182 & 48 & 49047448 \\
\hline
\end{tabular}

Sources : Données officielles compilées par l'auteur pour chaque pays.

Jusqu'au début des années 1990, les politiques migratoires et les discours sur l'immigration dans la sphère publique ont été largement dominés par une rhétorique économiste. Les discours officiels dans le Golfe ont tenté de faire croire à l'effacement du politique dans l'organisation de l'immigration, au profit des mécanismes de l'offre et de la demande, gérés par le marché et ses institutions (firmes, chambres de commerce, etc.). Ainsi, concernant les politiques internationales d'importation de main-d'œuvre et la sélection des migrants, un responsable de Chambre de commerce déclare :

"Le gouvernement ne voulait pas entrer dans des relations politiques avec les pays d'émigration. Les chambres de commerce et les comités de recrutement étaient les partenaires des gouvernements étrangers de sorte que le gouvernement saoudien n'était impliqué ni légalement ni politiquement dans l'immigration $»^{5}$.

L'importation de main-d'œuvre comme phénomène purement " économique ", temporaire, et sans conséquence sociale, se manifeste dans le refus symbolique d'utiliser le terme d' « immigré " :

"Nous n'utilisons pas le terme "migrants", nous utilisons le terme "expatriés" ou

"travailleurs étrangers". Le terme "migration" donne l'impression que les gens vont acquérir la nationalité. Nous préférons l'idée de "travailleur sous contrat", qui implique un

5 Entretien avec le secrétaire général de la Chambre de commerce de Djedda, 4 février 2006. 
calendrier et une durée de séjour définie. [...] C'est un aspect très important. Ces gens sont des travailleurs sous contrat temporaire, à la fin de leur contrat, ils rentrent chez eux ${ }^{6}$.

Cet effacement de l'État relève largement du mythe politique (Thiollet, 2010a et 2010b). Pour autant, la rhétorique de dépolitisation et de privatisation de l'immigration constitue un aspect clef du traitement politique de la question migratoire dans les pays du Golfe jusqu'au début des années 1990. Elle est fondée sur un " déni d'immigration".

\section{Politisation des migrations : crises et continuités depuis les années 1990}

Objet souvent présenté comme "infra politique " dans les monarchies du Golfe, la migration est en réalité un enjeu dans le champ politique notamment au tout début des années 1990, avec le renforcement des pratiques de contrôle des mouvements de populations et des communautés immigrées dans un contexte d'instabilité régionale. Des crises intermittentes constituent des moments de cristallisation d'une tendance institutionnelle : la " crise migratoire " de 1991 au moment de la guerre du Golfe (Stanton Russell, 1992 ; Thiollet, 2011) et la réaction des États de la région aux printemps arabes en 2011.

Dans un contexte de réduction des revenus pétroliers, à la fin des années 1980 , le pouvoir saoudien commence à affirmer sa volonté de réduire le volume de l'immigration en promouvant l'intégration des nationaux sur le marché du travail, la saoudisation de l'emploi. L'objectif est de conquérir une autonomie politique et économique par rapport à des voisins arabes pourvoyeurs de main-d'œuvre, d'abaisser le coût du travail', mais aussi de pallier l'apparition du chômage et la faillite sociale de modèles de développement économique extensifs. La volonté de limiter l'immigration, de nationaliser les marchés du travail est mise au cœur des discours sur l'immigration dans les pays du Golfe. Pourtant, elle n'a que peu ou pas de résultats effectifs, notamment du fait de la résistance d'un marché du travail très consommateur de main-d'œuvre immigrée. L'évaluation des politiques de saoudisation notamment a donné lieu à un constat d'échec (Hertog, 2012) : le secteur public absorbe la majorité des nouveaux entrants nationaux, les travailleurs étrangers, plus compétitifs et en terme de salaire et de qualification, occupent le secteur privé. Malgré I'inefficacité de ces politiques, l'immigration a clairement émergé comme question de politique intérieure et de " sécurité nationale " dans les années 1990, alors que le sujet était absent des débats publics dans la période précédente.

La guerre du Golfe en 1990-1991 correspond à un moment aigu de politisation des migrations : la dépendance des économies et la fragilité politique des États du Golfe sont révélées de manière brutale. Non seulement une partie des immigrants présents au Koweït en 1991 fuit devant I'arrivée des troupes irakiennes,

\footnotetext{
6 Entretien avec le vice-ministre de la Planification et du Développement, ministère du Travail et des Affaires sociales, Riyad, 27 janvier 2006.

7 Le changement de "stratégie d'importation " et le choix de la main-d'œuvre d'origine asiatique (Asie du Sud-Est, sous-continent indien) relèvent autant de logiques économiques (abaissement des coûts salariaux, flexibilité plus grande de la main d'œuvre et meilleure adéquation aux besoins du marché du travail en matière de qualification) que d'arbitrage de politiques intérieure et extérieure (Okruhlik et Conge, 1997 ; Thiollet, 2011).
} 
mais deux catégories de migrants arabes, les Yéménites et les Palestiniens sont ciblés par des campagnes brutales d'expulsions : 300000 Palestiniens sont expulsés du Koweït, 100000 d'Arabie saoudite (Leveau, 2003). 731800 Yéménites, selon le Central Statistical Bureau de Sanaa, sont expulsés d'Arabie suite au soutien implicite apporté par le gouvernement yéménite à l'invasion du Koweït par Saddam Hussein, alors qu'ils représentaient la plus grande communauté d'expatriés du pays, totalisant $27 \%$ des travailleurs étrangers (Thiollet, 2011). Au Koweït, une importante et prospère communauté palestinienne installée depuis les années 1950 devient la cible d'expulsions de politiques anti-immigration (visas ou permis de résidence non renouvelés, expulsions, licenciement, etc.) en réponse au soutien apporté par I'OLP à Saddam Hussein (Zelkovitz, 2014). Cet épisode attise une méfiance tenace à l'égard de l'immigration pourtant nécessaire au fonctionnement d'économies presque intégralement dépendantes de la main-d'œuvre immigrée. En retour, la crise de 1991 révèle la fragilité de la situation des migrants dans les pays du Golfe : privés de statut pérenne, ils font l'objet de mesures de rétorsion brutales et massives au nom de "la raison d'État " ou de la " sécurité nationale ".

Quelle forme institutionnelle prend cette politisation ? Elle s'incarne sous les auspices d'une gestion sécuritaire de la migration et notamment dans le transfert des prérogatives de gestion des flux et des résidents aux ministères de I'Intérieur des pays du Golfe (Lori, 2011). Elle se poursuit avec le développement de procédures d'enregistrement des migrants, et plus tard, de la biométrisation du contrôle de l'immigration (en 2006 pour l'Arabie saoudite).

Au début des années 1990, le ministère de I'Intérieur saoudien et son puissant ministre, le prince Na'ef, ministre de I'Intérieur depuis 1975, s'empare de la problématique migratoire. Le ministère de I'Intérieur et particulièrement le service de l'immigration et des passeports sont la clef de voûte du contrôle étatique de I'immigration. À partir de 1997, I'autorisation de délivrer un visa de visite ou de travail par les représentants du royaume à l'étranger doit obtenir l'accord du service de l'immigration et des passeports et les frais de visa doivent être payés avant émission du document par l'ambassade ou le consulat étranger ${ }^{8}$. La création du Conseil de la main-d'œuvre en 1990 marque la prise en main de la politique de saoudisation par le prince $\mathrm{Na}^{\prime}$ ef qui pilote les reformes menées au début des années 1990 avec un mandat aussi technique que politique : la saoudisation doit mobiliser la population autour d'un projet national pour remédier aux dissensions apparues avec la guerre du Golfe et la montée de l'opposition à la monarchie.

Au début des années 1990, le ministre tente de réformer la kafala9 ${ }^{9}$ le système de sponsor qui régule et contrôle la mobilité professionnelle et géographique

\footnotetext{
8 Décret du Conseil des ministres n 90 du14/5/1418 (17 septembre 1997) (traduit de l'arabe).

9 La kafâla est présentée comme un mode de contrôle de l'entrée, de la résidence et de la mobilité professionnelle et géographique des étrangers. Le garant ou kafîl est rémunéré par le migrant pour les services bureaucratiques qu'il fournit (permis de résidence, visas de sortie). Le coût de ce service peut varier, sur un marché partiellement informel, avec la nationalité, la qualification, le genre voire la religion du migrant. Pour de nombreux analystes, il constitue une forme d'exploitation ou de " rente migratoire " dont bénéficient les citoyens et un marqueur de hiérarchie et de différenciation entre nationaux et étrangers (Ibrahim, 1982 ; Longva, 1999).
} 
des migrants. Souvent considéré à tort comme un pur instrument de contrôle des migrants, la kafala constitue en réalité un point de tension entre les prérogatives de contrôle de l'État et celles du secteur privé : employeurs, recruteurs et kafill-s permettent à la marge d'un système public peu efficace de contourner les contraintes du droit du travail soit pour mieux exploiter les migrants (Murray, 2012) soit, plus rarement, pour leur accorder plus de flexibilité (en changeant d'employeur, mais pas de sponsor, ou en faisant venir leur famille). Le ministre de I'Intérieur, le prince Na'ef, multiplie les offensives pour " reprendre la main " sur le contrôle de l'immigration par les sponsors. En 1994, un décret du Conseil des ministres reprend la question de l'immigration clandestine et réaffirme la responsabilité des kafîl-s dans le contrôle de la régularité du séjour de leurs employés ${ }^{10}$. Le durcissement de la loi se traduit par la programmation légale de grandes entreprises de contrôle, d'incarcération et d'expulsion des étrangers en situation irrégulière. Le décret $n^{\circ} 30$ de 1994 met par exemple en place un système périodique de rafles ${ }^{11}$.

À partir de 2000, les enjeux sécuritaires et la guerre contre le terrorisme s'imposent à l'agenda politique et la question de la saoudisation de l'emploi perd de son importance. Le Human Resources Development Fund (HRDF), placé sous l'autorité du ministère du Travail, remplace le Conseil de la main-d'œuvre. Son poids politique, privé de la tutelle du prince $\mathrm{Na}^{\prime}$ ef est faible et le fonds se concentre initialement sur le financement de la formation ${ }^{12}$. Au même moment, la question migratoire disparaît des priorités de l'agenda politique, au profit d'autres enjeux sécuritaires (terrorisme), mais reste l'objet de réformes plus ou moins discrètes et réapparaît avec la " crise " politique générée par les printemps arabes.

En 2011, le ministère du Travail lance une nouvelle offensive sur les migrations et le travail. Avec Adel Faqih, ingénieur issu du secteur privé, ancien maire de Djedda et ministre du Travail d'août 2010 à avril 2015, soutenu par le roi Abdallah, le HRDF prend de l'importance dans le champ politique. L'affaiblissement de la kafala est inclus dans la réforme du marché du travail, initiée en septembre 2011, avec le lancement du nouveau programme de saoudisation appelé Nitaqat. La mobilité des travailleurs étrangers devient possible sans l'accord de leur employeur ou de leur kafil mais avec celui du ministère du Travail grâce au système informatique de contrôle de la mobilité appelé "Wafeed ": les travailleurs étrangers d'une entreprise qui compte moins de $50 \%$ de travailleurs saoudiens peuvent quitter leur emploi librement sans autorisation de leur sponsor ou de leur employeur. Sa mise en œuvre reste, bien entendu, difficile à évaluer.

10 Décret n³0 1/3/1415 (8 août 1994), Conseil des ministres, " Règlementation pour remédier à la situation des étrangers en situation irrégulière ou provisoire dans le royaume " (traduit de l'arabe).

11 lbid., " Décret pour remédier à la situation des étrangers en situation irrégulière ou provisoire dans le royaume " (traduit de I'arabe).

12 Voir le site du HRDF : https://www.hrdf.org.sa/ 


\section{Migration et politiques de saoudisation : une ingénierie sociale contre-révolutionnaire}

De nombreux observateurs ont considéré en 2011 que la redistribution de l'argent du pétrole par l'État, c'est-à-dire la famille royale, dans le contexte révolutionnaire devait " acheter la paix sociale ". La politique saoudienne de cooptation s'est en effet traduite par une augmentation des dépenses publiques ${ }^{13}$ d'environ $15 \%$ entre 2010 et 2011 qui ont servi de rappel de l'allégeance collective de la nation à l'État rentier. Les 23 février et 18 mars 2011, le roi Abdallah annonce le déblocage d'un premier volant de 130 milliards de dollars en aides sociales, incluant la mise en place de subventions pour l'accès au logement. La tendance est prolongée par le roi Salman en mars 2015, avec le changement de ministre du Logement, qui annonce des aides d'accès à la propriété pour 600000 familles et promet de construire 500000 unités de logements neufs ${ }^{14}$. Plusieurs centaines de millions de dollars sont aussi débloqués pour financer des institutions religieuses.

L'arsenal de politiques de réforme du marché du travail lancée en 2011 entre à la fois dans une démarche démagogique de promotion de la " nation saoudienne au travail ", d'achat de la paix sociale grâce à des politiques structurelles au cœur d'un projet de modernisation de l'économie saoudienne et la désignation de boucs émissaires (les immigrants). Les objectifs avoués de réduction du chômage des jeunes et des femmes et d'accroissement de la compétitivité des Saoudiens par rapport aux travailleurs immigrés sont en effet combinés avec un objectif de court terme d'achat de la paix sociale notamment auprès des classes populaires et des jeunes qualifiés.

\section{Nitaqat et Hafez (2011) : saoudisation et invention du chômage}

Nitaqat, littéralement "zone ", classe les entreprises privées en fonction de leur degré d'intégration des travailleurs saoudiens. Un système de pénalités et de récompenses attribuées par le ministère du Travail correspondant au taux de Saoudiens employés dans l'entreprise en fonction de la taille de l'entreprise (quatre classes sont distinguées) permet de créer des contraintes et des incitations à même de favoriser l'emploi des Saoudiens. Les entreprises classées Rouge et Jaune qui embauchent environ moins de $11 \%$ de Saoudiens (les seuils variant en fonction du nombre d'employés) n'ont plus la possibilité de faire venir de nouveaux travailleurs immigrés (et donc de demander des visas au ministère du Travail) ou de renouveler le visa de leurs travailleurs étrangers tandis que les entreprises classées Vert et Platinum peuvent renouveler ou faire venir de nouveaux travailleurs immigrés. Nitaqat introduit pour les travailleurs des entreprises classées Rouge puis Jaune la possibilité de quitter leur emploi pour aller travailler dans une entreprise Vert ou Platinum sans I'autorisation de leur employeur : c'est une première en matière de "liberté " des salariés

13 MacFarquhar Neil (2011) Saudi Cash is the Key to Quiet in the Kingdom, The International Herald Tribune (June 10, 2011), p. 4 ; MacFarquhar Neil (2011) Saudis Scramble in Bid to Contain Regional Unrest, The New YorkTimes (May 28, 2011), p. 1.

14 The National (2015) Saudi Arabia replaces housing minister after king vows to address shortage (March 12, 2015), [en ligne] consulté le 15/09/2015. URL : http://www.thenational. ae/world/middle-east/saudi-arabia-replaces-housing-minister-after-king-vows-to-addressshortage 
qui introduit une compétition entre les entreprises. Le poids de ces nouvelles régulations pèse largement sur les petites entreprises qui constituent l'immense majorité des 17000 entreprises classées Rouge.

II s'agit de remplacer les immigrants par les nationaux dans le secteur privé, afin de faire baisser le chômage. En 2014, les Saoudiens représentent, d'après les statistiques officielles, 5591563 personnes soient 46,9\% de la population active (de plus de quinze ans) et $44 \%$ de la population active occupée ${ }^{15}$. La fiabilité des statistiques produites par les organes officiels saoudiens reste discutable et les chiffres du chômage sont très probablement sous-estimés, en partie parce qu'ils ne prennent pas en considération le sous-emploi d'une grande partie de la population active saoudienne (Hertog, $2012: 14)^{16}$. Les différents rapports publiés par les organismes publics saoudiens indiquent une préoccupation croissante pour les chiffres du chômage et la répartition des Saoudiens sans emploi par âge, sexe et niveau d'études. Ainsi l'enquête sur le marché du travail de 2015 indique que 646854 actifs saoudiens sont sans emploi (soit 11,6\%), qu'une majorité sont des femmes et que 50 \% d'entre eux ont un diplôme d'études supérieures ${ }^{17}$. À terme, près de 2 millions de jeunes saoudiens ont entre quinze et dix-neuf ans en 2014, ce qui signifie qu'environ 400000 Saoudiens atteignent chaque année l'âge d'entrer dans la vie active. Le secteur public qui était la variable d'ajustement de l'État pour éviter le chômage et assurer la redistribution de la rente pétrolière à travers le fonctionnariat, semble avoir atteint sa limite avec entre 900000 et 1,8 million de fonctionnaires selon les sources publiques ou un rapport indépendant (Haykel et al., 2015 : 101-102). Seuls environ 800000 Saoudiens seraient employés dans le secteur privé formel.

La création d'un système d'assurance chômage pour les nationaux, nommé Hafez, en novembre 2011, permet à la fois d'identifier les populations hors du marché du travail (femmes, travailleurs du secteur informel, autres inactifs) et d'étendre le système de distribution de la rente pétrolière aux classes les plus populaires. Sur simple déclaration, les " chômeurs " reçoivent une allocation de 2000 riyals par mois. Dès janvier 2012, près d'1 million de personnes sont enregistrées, parmi lesquels essentiellement des femmes (à plus de $80 \%$ ). La création de Hafez répond ainsi aux objectifs de contrôle et de collecte d'information sur la population et d' " achat de la paix sociale " autant qu'aux objectifs avoués de réduction du chômage des jeunes et des femmes en âge de travailler.

La politique de l'emploi doit compenser la trop forte compétitivité de la main-d'œuvre expatriée, tant en matière de compétence (pour les segments les plus qualifiés) que du coût du travail (pour les segments les moins qualifiés). L'ingénierie financière déployée vise explicitement à renverser le désavantage " structurel " des Saoudiens sur le marché du travail en creusant les inégalités statutaires entre étrangers et Saoudiens. Alors que les vagues de saoudisation précédentes étaient fondées sur des politiques publiques répressives

15 Central Department Statistical Information, Saudi Arabia, Labour Force Survey (Document en arabe), [en ligne] consulté le 15/09/2015. URL : http://www.stats.gov.sa/

16 Voir les calculs de Hertog (2012 : 14) à partir d'un croisement des statistiques des différents organes publics saoudiens. II calcule qu'un actif saoudien sur quatre seulement serait " occupé " en 2007.

17 Central Department Statistical Information, Saudi Arabia, Labour Force Survey (Document en arabe), [en ligne] consulté le 15/09/2015. URL : http://www.stats.gov.sa/ 
(amendes), celle de 2011 s'appuie sur un large spectre d'instruments incluant incitations et répression. II s'agit d'éviter les stratégies d'évasion du secteur privé lors des précédentes vagues de saoudisation : la création de " sociétésécrans ", visant à permettre l'importation de main-d'œuvre au-delà des seuils autorisés en évitant les amendes, est particulièrement visée. Nombre d'interviewés dans le secteur privé, comme dans le secteur public, reconnaissent qu'à défaut de réellement promouvoir l'emploi des Saoudiens dans le secteur privé, la saoudisation jusqu'en 2011 avait généré la création d'un nombre important de " faux business ${ }^{18}$ pour permettre aux entreprises de contourner les réglementations. Parmi les quelques 200000 entreprises qui ont disparu des registres du système d'assurance sociale (GOSI) à partir de I'application du programme ${ }^{19}$, nombre d'entre elles sont de fait de toutes petites unités productives, des entreprises intégralement dépendantes de la main-d'œuvre étrangère qui ont dû fermer. Mais ces destructions concernent largement des sociétés-écrans, dont la fonction principale était de pouvoir produire des visas d'importation de maind'œuvre qui étaient ensuite " revendus " à d'autres entités.

\section{Taxer I’immigration pour subventionner I'emploi et financer le chômage}

Les politiques de subvention des emplois saoudiens dans le secteur privé sont généralisées dès 2011. L'État saoudien s'engage à subventionner jusqu'à $50 \%$ des salaires des employés saoudiens à travers le HRDF pour une durée qui peut aller jusqu'à quatre ans en fonction des efforts fournis par les entreprises en matière de saoudisation : à travers des " campagnes " successives, le HRDF va jusqu'à rembourser une partie des salaires des employés saoudiens aux entreprises qui sont bien " notées " dans le cadre du programme Nitaqat. Ces subventions sont alimentées entre autres par la taxation de l'immigration. À partir de 2013, une amende mensuelle de 200 riyals saoudiens est versée par l'employeur pour chaque employé qui dépasse le quota d'étrangers autorisé dans I'entreprise (moins de $50 \%)^{20}$. Par ailleurs, un montant fixe de frais administratifs liés à l'enregistrement en résidence des travailleurs étrangers (iqama) de 2500 riyals saoudiens est aussi payé par l'employeur directement au HRDF pour ces mêmes travailleurs étrangers "surnuméraires " dans le cadre de Nitaqat. II s'agit à court terme d'augmenter le coût du travail des étrangers dans les secteurs d'activités où les Saoudiens peuvent les remplacer et en même temps, d'abaisser artificiellement le coût du travail des Saoudiens, plus cher que celui des expatriés. En d'autres termes, il s'agit de contrer un des effets principaux de l'économie rentière, qui est la distorsion poussée à l'extrême du marché du travail. Le financement des salaires permet de baisser les résistances des employeurs à la saoudisation et fait espérer aux bureaucrates saoudiens, flanqués de consultants occidentaux de meilleurs résultats qu'au début des

18 Entretien avec un entrepreneur, Djeddah, janvier 2015.

19 Arab News (2014) Nitaqat: 200,000 firms closed down (5 August, 2014), [en ligne] consulté le 15/09/2015. URL : http://www.arabnews.com/news/611896

20 Entretiens au ministère du Travail, janvier 2015. 
années 1990. Comme l'indiquent des représentants du ministère du travail ${ }^{21}$ :

"Le coût du travail des Saoudiens va progressivement baisser et converger vers celui des expatriés pour les emplois qualifiés et très qualifiés. Nous prévoyons de faire décroître progressivement les subventions à mesure que le coût du travail des Saoudiens dans le secteur privé rejoint le prix du marché. Nous comptons sur le fait que les Saoudiens vont devenir plus compétents et plus compétitifs $»^{22}$.

La capacité de l'État saoudien à opérer ce " grand remplacement " (des étrangers par les Saoudiens) reste sujette à caution compte tenu des écarts de salaire, de formation, de productivité, etc. Elle présente néanmoins un discours et des dispositifs politiques " anti-immigration " très puissants : les besoins d'étrangers sur le marché du travail doivent s'affaiblir. Corrélé à des mesures technocratiques et financières, l'État saoudien déploie aussi des campagnes de fragilisation et de discrimination contre des catégories spécifiques d'étrangers, les migrants irréguliers.

\section{Expulser les étrangers : signification politique d'une discipline sociale}

L'offensive menée en Arabie saoudite à partir de 2011 contre l'immigration clandestine et les résidents étrangers en situation irrégulière prolonge une tendance préexistante et l'amplifie. Une campagne d'enregistrement et de fichage des expatriés est lancée dans le Royaume en novembre $2006^{23}$ pour maîtriser l'immigration clandestine et contrôler la durée de séjour des immigrants, notamment dans le cadre du pèlerinage. En 2006, le service de l'immigration et des passeports commence à conserver les fichiers des clandestins pour éviter les ré-émigrations : la criminalisation de l'immigration clandestine s'impose progressivement là où jusqu'alors, l'informalité était largement tolérée.

\section{" Régulariser " et amnistier : une " remise en ordre " du business migratoire ?}

La campagne de régularisation visant à rétablir la situation légale de certains étrangers a concerné près de 4 millions de personnes d'après les statistiques publiées par le ministère du Travail en $2013^{24}$ et jusqu'à 5,3 millions d'après la presse ${ }^{25}$ soit la moitié des étrangers officiellement présents sur le territoire

\footnotetext{
21 De manière symptomatique, les emplois les moins qualifiés sont exclus de cette politique de substitution: les responsables de la réforme au sein du ministère du Travail comme du HRDF précisent que les deux extrémités du marché du travail, les emplois non qualifiés et les emplois les plus qualifiés resteront occupés par les étrangers. Entretiens au ministère du Travail, janvier 2015.

22 Entretiens au Human Resource Development Fund, janvier 2015.

23 Shaikh Habib (2006) Random finger-printing of expats in Saudi from today (4 November, 2006), Al-Khaleej Times.

24 Les données concernant les changements de statut juridique et changement de kafîl sont compilées de 2010 à 2013 sur le site du ministère du Travail. Cf. http://portal.mol. gov.sa/ar/Statistics/Pages/default.aspx?m=2 et http://portal.mol.gov.sa/en/News/Pages/4 million_corrective_procedures_recorded_up_to_27_Shaba\%E2\%80\%99n_1434H.aspx

25 " More than 1.5 million workers transferred their sponsorship to new employers and 1.3 million changed their work description, while 2.5 million changed their residence permits " (http://www.arabnews.com/news/463365).
} 
saoudien. L'offensive de 2011 touche particulièrement une pratique illégale, mais néanmoins courante de l'immigration, celle des "free visas " : les travailleurs sont " importés " par un recruteur à l'aide d'un visa en règle et bénéficient de la tutelle d'un kafil mais n'ont pas de contrat de travail. Cette pratique permet la création d'un volant de main-d'œuvre disponible qui absorbe les fluctuations de la demande du marché du travail. Elle est notablement liée aux besoins du secteur du bâtiment, des services, de l'ensemble des emplois non qualifiés précaires. Elle repose sur l'existence d'entreprises "écrans " qui déclarent une activité fictive pour obtenir du ministère de I'Intérieur et du Travail la possibilité $d^{\prime}$ " importer des travailleurs " qui sont ensuite " revendus " à d'autres entreprises sur une base journalière, hebdomadaire ou mensuelle, ou qui partent travailler avec ou sans contrat de travail pour un autre employeur en payant à l'entreprise qui les a fait entrer dans le pays, et qui reste leur sponsor, une somme fixe annuelle ou une part de leur salaire. Ces sociétés constituent l'épine dorsale du business de la migration, véritable secteur d'activité qui comprend aussi des entreprises de recrutement positionnées entre les pays d'origine et les pays d'accueil du Golfe, un secteur extrêmement lucratif, qui s'arroge une " rente migratoire " en opérant aux marges de la législation saoudienne.

Les vagues d'expulsion sont précédées de périodes " d'amnistie " qui permettent d'identifier les populations concernées et offrent une occasion de régularisation sélective d'immigrés. Les politiques d'amnistie permettent aux migrants en situation irrégulière d'être déportés sans poursuites pénales (et partant, d'envisager d'y revenir légalement) ou de régulariser leur situation sur place, ceci en fonction du type d'infraction constatée. Le soutien des ambassades et des consulats à leurs ressortissants est révélateur de la politique diasporique des pays d'origine : les ambassades indiennes ont par exemple vigoureusement soutenu leurs ressortissants et même obtenu la prolongation de la période d'amnistie entre juillet et octobre 2013.

Ces périodes d'amnistie sont l'occasion d'un intense trafic de services administratifs et de corruption permettant aux migrants et à leur famille de régulariser leur situation par exemple en validant leur affiliation à un nouveau kafill, ou en signant un contrat de travail alors qu'ils exerçaient de manière informelle jusque-là. Au coût des procédures administratives légales impliquées, sont ajoutés les frais d'une corruption généralisée qui pèse sur l'ensemble du dispositif de gestion de la migration ${ }^{26}$. Le coût de la régularisation pour les immigrants est très élevé : I'achat de la bienveillance des différents niveaux de l'administration concernés par les procédures de régularisation se superpose au coût officiel des procédures ; la menace d'expulsion met les employés en situation difficile vis-à-vis de leurs employeurs, les prive de toute marge de négociation en matière de droits ou de salaire.

\section{Expulsions et campagnes de régularisation}

Les expulsions font partie des pratiques routinières de gestion de l'immigration clandestine. L'Arabie mêle une tolérance habituelle du secteur informel et des travailleurs étrangers en situation irrégulière avec des accès de répression massive. Les expulsions de travailleurs en situation irrégulière commencent

26 Entretien avec un migrant pakistanais, à Djedda, en janvier 2015. 
à la fin des années 1970 et très vite atteignent un rythme soutenu d'au moins 300000 personnes jusqu'à 700000 par an selon certaines sources ${ }^{27}$. Jusqu'au début des années 2000, I'usage et la pratique des expulsions en Arabie saoudite reflètent un type particulier de gestion des flux de main-d'œuvre. La circulation des travailleurs - turn-over - y est assurée par un régime plutôt libéral d'entrée sur le territoire et une forte proportion d'immigration clandestine ou un faible contrôle de la résidence qui permet à de nombreux travailleurs et à leur famille de résider sur le territoire dans des situations de semi-irrégularité, avec des visas de pèlerinage ou de travail périmés (overstayers), des permis de travail obsolètes, grâce souvent, à la garantie de kafil de complaisance (leur sponsor acceptant de garantir leur séjour même s'ils n'ont pas de contrat de travail en règle). Le cas des étrangers entrés en Arabie avec un visa de pèlerinage et resté pour travailler durant quelques mois est exemplaire : leurs stratégies migratoires incluaient souvent l'expulsion comme moyen de rentrer gratuitement " au pays ".

La faiblesse des contrôles, la possibilité de corrompre les policiers chargés d'enregistrer des migrants irréguliers et le faible degré de formalisme dans l'emploi, notamment peu qualifié, ont fait de l'immigration irrégulière une variable d'ajustement pour le marché du travail saoudien.

Avec la campagne de régularisation de 2011, l'État saoudien semble rompre avec les pratiques et les dynamiques informelles. Elle concerne les migrants " sans papiers ", mais aussi ceux qui ont changé de sponsors, ceux dont le permis de séjour est périmé, ceux dont l'intitulé du contrat de travail ne correspond plus à ce qui est indiqué sur leur permis de résidence (iqama), ceux dont l'emploi ne correspond pas à l'intitulé de leur permis de travail, etc. La moindre irrégularité peut être source de révocation du permis de résidence. Selon les autorités saoudiennes, la campagne aurait permis la " régularisation " d'environ 5 millions de personnes, dont 1 million auraient quitté le pays durant la période d'amnistie de 2013. Entre novembre 2013 (fin de la période d'amnistie) et avril 2014, le ministère de I'Intérieur saoudien annonce l'expulsion de 427000 personnes. Les expulsions sont pratiquées de manière clairement discriminatoire : elles visent notamment les catégories les plus pauvres de la population étrangère, les travailleurs les moins qualifiés. Dans un contexte de segmentation du marché du travail par nationalités, cela revient à viser certaines nationalités plutôt que d'autres. Les expulsions touchent donc massivement les populations éthiopiennes, somaliennes ou yéménites en situation irrégulière et épargnent l'immigration occidentale ou indienne qualifiée. Entre juin 2013 et novembre 2014, I'OIM a pu compter 613743 Yéménites déportés à travers la frontière sud du pays, au point de passage d'al Tuwal ${ }^{28}$. Les conséquences pour le Yémen avoisinent celles du choc migratoire de 1991 en matière d'appauvrissement et de chômage dans un contexte de guerre civile ${ }^{29}$. Entre novembre 2013 et janvier 2015, ce sont presque 2 millions de personnes qui auraient été déportées, le

27 Arab News (2002) Kingdom deports 700,000 illegals annually: Official (May 21, 2002).

28 Detained, Beaten, Deported. Saudi Abuses against Migrants during Mass

Expulsions (10 mai 2015), [en ligne] consulté le 13/06/2015. URL : https://www.hrw.org/ report/2015/05/10/detained-beaten-deported/saudi-abuses-against-migrants-during-massexpulsions

29 http://www.aljazeera.com/indepth/features/2013/05/20135137243618120.html 
ministère $\mathrm{n}^{\prime}$ ayant pas publié de statistiques officielles ${ }^{30}$.

Des policiers, lors de barrages routiers mis en place par le ministère de l'Intérieur dans le Hedjaz et autour de la Mecque, vérifient les papiers des étrangers pour traquer les "faux pèlerins ". Des rafles sont organisées, ciblant particulièrement les quartiers populaires des grandes villes, comme Bani Malik à Djedda, Manfuha, au sud de Riyad. Les travailleurs peu qualifiés, du secteur du bâtiment notamment, sont touchés par les rafles dans leurs lieux de vie et sur leurs lieux de travail ou sur les axes routiers proches des chantiers : souvent embauchés à la journée, ils sont en fait en situation irrégulière, sans iqama, permis de résidence et sans kafîl, même si leur visa est régulier. Le secteur des petites entreprises aux emplois peu qualifiés est lui aussi touché : les étrangers y travaillent souvent sous des statuts irréguliers ou seulement partiellement réguliers, détenteurs de "free visa " ou entrés dans le pays grâce à un visa de pèlerinage.

De fait, les expulsions sont à la fois un signe de la brutalisation globale des rapports sociaux et de l'augmentation des inégalités comme le montre Sassen (2014). Mais elles constituent surtout dans le cas saoudien, un mode de subordination des étrangers par l'État, subordination fondée sur le risque permanent (De Genova et Peutz, 2010). À ce titre, les tentatives d'étatisation de la gestion des migrations par des politiques d'immigration massive et des expulsions fréquentes constituent une forme de "discipline sociale " (Foucault, 1999), qui combine exclusion et normalisation des " mécanismes " qui intègrent et contrôlent les étrangers. Dans une société et une économie où l'État se veut en général peu présent et où les acteurs privés (recruteurs, employeurs, kafîl-s) entrent en compétition avec lui, les expulsions fonctionnent comme des rappels de l'existence de la puissance "publique " et de sa fonction de régulation. Pourtant les entretiens menés dans l'administration publique ont montré que les expulsions n'ont pas vocation à " empêcher l'immigration " ${ }^{31}$, mais plutôt à assurer un contrôle accru de l'État sur les immigrants. Pour les immigrés et pour leurs employeurs, cette offensive a coûté cher et elle a contribué à fragiliser un peu plus la position des premiers.

Dans les années 1990, les politiques anti-immigration n'avaient pas fait baisser le taux d'immigration nette, ni n'avaient réduit la part de la population étrangère dans la population globale comme l'indique la figure 1 . On peut supposer que les tendances seront similaires à moyen terme, quand bien même le turn-over migratoire serait plus intense et les effectifs d'entrées et de sorties de migrants plus élevés. Les entretiens menés au sein du ministère du Travail ou du ministère de I'Intérieur indiquent clairement que le but de la politique saoudienne n'est pas de faire cesser l'immigration de main-d'œuvre, mais de mieux la sélectionner et la contrôler ${ }^{32}$.

30 http://www.ft.com/intl/cms/s/0/6b49c0fa-5d9d-11e3-b3e8-00144feabdc0. html\#axzz3x7pvcyCh

31 Entretien, ministère du Travail, 11 janvier 2015.

32 Entretiens, ministère du Travail, janvier 2014 et janvier 2015. 


\section{Conclusion : migration et changement social}

La focalisation des États sur le contrôle politique de leur population et le contrôle social et économique de leurs populations étrangères sert de révélateur aux fragilités profondes des systèmes de gouvernement et de l'économie politique des monarchies golfiennes. Certes, la répression des mouvements d'opposition politique en Arabie saoudite et à Bahreïn a fait taire momentanément les foyers de révolte. Plus profondément, les monarchies et en particulier l'Arabie saoudite, commencent à s'inquiéter de déséquilibres structurels de leurs sociétés qui pourraient se révéler incontrôlables. En Arabie saoudite, pour éviter l'émergence d'une génération mécontente qui remettrait en cause le pacte socio-économique et politique de l'État rentier, le gouvernement se livre à un exercice d'ingénierie socio-économique où les migrants sont la variable d'ajustement d'une construction nationale encore inachevée. Or, les politiques anti-immigration et anti-intégration connaissent un double échec. L'échec des premières est aisément constaté. Malgré une baisse temporaire de l'immigration dans les années 1990, celle-ci a repris dans les années 2000. Celui des secondes se manifeste par le phénomène d'installation des communautés immigrées qu'elles soient arabes ou asiatiques, révélé statistiquement par l'allongement de la durée de séjour ainsi que l'existence de deuxièmes et troisièmes générations d'étrangers nés dans le Golfe (Thiollet, 2010b). Certains éléments quantitatifs (démographie des communautés immigrées) et qualitatifs (Hanieh, 2009 ; Vora, 2013) permettent de conclure à une installation de fait, notamment à travers les interactions sociales existant entre immigrés et nationaux, ce malgré les dispositifs anti-intégration (Thiollet, 2010b). De fait, pour reprendre l'expression triviale de Martin, il semble qu'il n'y ait « rien de plus permanent qu'un travailleur temporaire " (Martin et Miller, $1980: 315$ ).

Plus qu'un changement de régime, c'est donc, une révolution sociale qui peut s'opérer dans les monarchies du Golfe en lien avec l'immigration massive. L'émergence de sociétés multiculturelles de fait, mais inégalitaires en droit, de communautés de migrants intégrées de fait, mais exclues en droit. L'Arabie saoudite déploie un arsenal de politiques migratoires et de politiques sociales qui visent à transformer les équilibres du marché du travail et de la démographie, à éviter la " révolution sociale " qu'implique la pérennisation de son modèle migratoire. On peut se demander néanmoins si malgré la radicalité et la brutalité des mesures, leur coût exorbitant, tant financier qu'humain, cette ingénierie des politiques publiques en temps de crise peut renverser des (des-) équilibres démographiques durables qui sont aussi des réalités sociales. Les ferments de I'hypothétique "révolution sociale " dans les monarchies du Golfe sont de fait indissociablement liés à la question migratoire : loin d'être simplement des instruments de " développement " (Glick Schiller et Faist, 2009), ces dynamiques de changement social (Castles, 2001) échappent aux ingénieurs pour reprendre la métaphore de Tilly. 


\section{Références bibliographiques}

Beaugé Gilbert et Roussillon Alain (1988) Le migrant et son double : migration et unité arabe, Paris, PUBLISUD, 191 p.

Brand Laurie Ann (1988) Palestinians in the Arab World: Institution Building and the Search for State, New York, Columbia University Press, 286 p.

Castles Stephen (2001) Studying Social Transformation, International Political Science Review/Revue internationale de science politique, 22 (1), pp. 13-32.

Chalcraft John (2011) Migration and popular protest in the Arabian Peninsula and the Gulf in the 1950s and 1960s, International Labor and Working Class History, 79 (1), pp. 28-47.

Coates Ulrichsen Kristian (2013) Bahrain's Uprising: Regional Dimensions and International Consequences, Stability. International Journal of Security \& Development, 2 (1), 14, pp. 1-12.

De Bel-Air Françoise (2012) Printemps Arabes et gestion des migrations dans les pays du Golfe : vers une réforme politique, Migrations société, 4 (143), pp. 87-103.

De Genova Nicholas P. and Peutz Nathalie (Eds.) (2010) The Deportation Regime: Sovereignty, Space, and the Freedom of Movement, Duke University press, $520 \mathrm{p}$.

Fargues Philippe (2000) Générations Arabes, l'alchimie du nombre, Paris, Fayard, $349 \mathrm{p}$.

Foucault Michel (1999) Cours au Collège, de France 1974-1975. "Les anormaux ", Gallimard Le Seuil, 356 p. (coll. Hautes Études).

Gause Gregory (2011) Is Saudi Arabia really counter-revolutionary?, Foreign Policy, [online] last checked on 11/06/2015. URL: http://foreignpolicy.com/2011/08/09/ is-saudi-arabia-really-counter-revolutionary/

Glick Schiller Nina and Faist Thomas (2009) Migration, Development, and Social Transformation, Social Analysis, 53 (3), pp. 1-13.

Hanieh Adam (2009) Temporary Migrant Labour and the Spatial Structuring of Class in the Gulf Cooperation Council, Presentation to the 3rd International Initiative Promotion and Political Economy (IIPPE) Workshop, Ankara, Turkey 14-15 September, [online] last checked on 28/09/2015. URL: http://spectrumjournal.net/wp-content/uploads/2014/05/26-41-1-SM.pdf

Haykel Bernard, Lacroix Stéphane and HegghammerThomas (2015) Saudi Arabia in Transition: Insights on Social, Political, Economic and Religious Changes, Cambridge, Cambridge University press, 357 p.

Hertog Steffen (2012) A comparative assessment of labor market nationalization policies in the GCC, in Steffen Hertog Ed., National employment, migration and education in the GCC. The GulfRegion: economic development and diversification, Berlin, Gerlach Press, Cambridge [online] last checked on 28/09/2015. URL: http:// eprints.Ise.ac.uk/46746/1/A\%20comparative\%20assessment $\% 20$ of\%20labor $\% 20$ market\%20nationalization\%20policies\%20in\%20the\%20GCC\%28lsero\%29.pdf

Hoffman Michael and Amaney Jamal (2012) The Youth and the Arab Spring: Cohort Differences and Similarities, Middle East Law and Governance, 4, pp. 168-188. 
Ibrahim Saad Eddin (1982) Oil, Migration and the New Arab Social Order, in Maclom H. Kerr and Yassin El Sayyed Eds., Rich and Poor States in the Middle East: Egypt and the New Arab Social Order, Boulder Colorado, Westview Press, pp. 17-69.

Kamrava Mehran (2012) The Arab Spring and the Saudi-Led Counterrevolution, Orbis, 56 (1), pp. 96-104.

Kanna Ahmed (2012) A politics of non recognition? Biopolitics of Arab Gulf worker protests in the year of uprisings, Interface, a journal for and about social movements, 4 (1), pp. 146-164.

Lacroix Stéphane (2014) Saudi Islamists and the Arab Spring, Kuwait Programme on Development, Governance and Globalisation in the Gulf States, [online] last checked on 28/09/2015. URL: http://eprints.Ise.ac.uk/56725/1/Lacroix_SaudiIslamists-and-theArab-Spring_2014.pdf

Leveau Rémy (2003) Imaginaires et conflits dans I'espace euro-méditerranéen, Esprit, 12, pp. 144-150.

Levitt Peggy (1998) Social Remittance: Migration Driven Local Level Forms of Cultural Diffusion, International Migration Review, 32 (4), pp. 926-948.

Longva Anh Nga (2005) Neither Autocracy Nor Democracy but Ethnocracy: Citizens, Expatriates and the Socio-Political System in Kuwait, in Paul Dresch and James Piscatori Eds., Monarchies and Nations: Globalisation and Identity in the Arab States of the Gulf, New York, IB Tauris, pp. 114-135.

Longva Anh Nga (1999) Keeping Migrant Workers in Check: The Kafala System in the Gulf, Middle East Report, 211, Trafficking and Transiting: New Perspectives on Labor Migration, pp. 20-22.

Lori Noora (2011) National Security and the Management of Migrant Labor: A Case Study of the United Arab Emirates, Asian \& Pacific Migration Journal, $20(3 / 4)$, pp. 315-337.

Martin Philipp and Mark Miller Mark (1980) Guestworkers: Lessons from Western Europe, Industrial and Labor Relations Review, 33 (3), pp. 315-330.

Matthiesen Toby (2014) Migration, Minorities, and Radical Networks: Labour Movements and Opposition Groups in Saudi Arabia, 1950-1975, IRSH, 59, pp. 473-504.

Murray Heather (2012) Hope for Reform Springs Eternal: How the Sponsorship System, Domestic Laws andTraditional Customs Fail to Protect Migrant Domestic Workers in GCC Countries, Cornell International Law Journal, 45, pp. 461-485.

Okruhlik Gwenn (1999) Excluded Essentials: The Politics of Ethnicity, Oil, and Citizenship in Gwen Moore and J. Allen Whitt Dir., The Global Color Line. Racial and Ethnic Inequality and Struggle from a Global Perspective, vol. 6, Emerald Group Publishing, $400 \mathrm{p}$.

Okruhlik Gwenn and Conge Patrick (1997) National Autonomy, Labor Migration and Political Crisis: Yemen ans Saudi Arabia, Middle East Journal, 51 (4), pp. 554-565.

Sassen Saskia (2014) Expulsions. Brutality and Complexity in the Global Economy, Cambridge, Harvard University Press, 304 p. 
Seccombe lan and Richard I. Lawless (1986) Foreign Worker Dependence in the Gulf, and the International Oil Companies: 1910-50, International Migration Review, 20 (3), pp. 548-574.

Shafeeq M. Najeeb, Mason Jessica, Seybolt Taylor and DeLuca Kristin (2014) Are student protests in Arab states caused by economic and political grievances? Empirical evidence from the 2006-07 Arab Barometer, Peabody Journal of Education, 89 (1), pp. 141-158.

Shafik Nemat (1999) Labor Migration and Economic Integration in the Middle East, in Michael C. Hudson Ed., The Middle East Dilemma, Washington, DC, Columbia University Press, pp. 279-98.

Skocpol Theda (1979) States and social revolutions. A comparative analysis of France, Russia and China, Cambridge, Cambridge university press, 350 p.

Stanton Russell Sharon (1992) International Migration and PoliticalTurmoil in the Middle East, Population and Development Review, 18, pp. 719-727.

Steinberg Guido (2014) Leading the Counter-Revolution: Saudi Arabia and the Arab Spring, Stiftung Wissenschaft und Politik, Berlin, German Institute for International and SecurityAffairs, [online] last checked on 12/08/2015. URL: http:// www.swp-berlin.org/fileadmin/contents/products/research_papers/2014_RP07_ sbg.pdf

Thiollet Hélène (2013) Migration, exil et révolutions, in Alain Dieckhoff et Frédéric Charillon Dir., Mondes Émergents, Paris, La Documentation française, pp. 133-146.

Thiollet Hélène (2011) Migration as Diplomacy: Labour Migrants, Refugees and Arab regional politics in the oil rich countries, International Labor and Working Class History, 79, pp. 103-121.

Thiollet Hélène (2010a) Migration and National Identity in Saudi Arabia, The 2010 Gulf Research Meeting, University of Cambridge, July 7-10.

Thiollet Hélène (2010b) Nationalisme ordinaire et nationalisme d'État en Arabie Saoudite : la Nation saoudienne et ses immigrés, Raisons politiques, 37, pp. 89-102.

Tilly Charles (1973) Revolutions and collective violence, CRSO Working Paper 83, University of Michigan, [online]. URL: http://deepblue.lib.umich.edu/bitstream/ handle/2027.42/50863/83.pdf

Vitalis Robert (2007) America's Kingdom: Mythmaking on the Saudi Oil Frontier, California, Stanford University Press, 304 p.

Vora Neha (2013) Impossible Citizens: Dubai's Indian Diaspora, Duke University Press, 264 p.

Weyland Kurt (2012) The Arab Spring: Why the Surprising Similarities with the Revolutionary Wave of 1848?, Perspective on Politics, 10 (4), pp. 917-934.

Winckler Onn (1997) The Immigration Policy of the Gulf Cooperation Council (GCC) States, Middle Eastern Studies, 33 (3), pp. 480-493.

Zelkovitz Ido (2014) A Paradise Lost? The Rise and Fall of the Palestinian Community in Kuwait, Middle Eastern Studies, 50 (1), pp. 86-99. 


\section{Hélène Thiollet}

\section{Migration et (contre)révolution dans le Golfe : politiques migratoires et politiques de I'emploi en Arabie saoudite}

Alors que des révolutions politiques mettaient à mal les régimes autoritaires du Moyen-Orient, les monarchies du Golfe et, en particulier, I'Arabie saoudite, ont déployé à l'échelle nationale et internationale des politiques " contre-révolutionnaires ". Au-delà de la répression de l'opposition politique, l'Arabie saoudite se lance dans une campagne de réforme de l'immigration et de l'emploi qui a vocation à réduire les facteurs structurels de mécontentement économique et social de sa population et "prévenir " une révolution. Véritable ingénierie sociale, ces politiques s'inscrivent dans une tendance lourde de politisation de l'immigration depuis le début des années 1990 et de construction de la " menace migratoire " comme risque existentiel pour la pérennité de la nation saoudienne. On avance dans cet article l'idée qu'au-delà du changement de régime c'est bien le risque d'une "révolution sociale ", selon les termes de Theda Skocpol, qui veut être évité en réaffirmant les rapports de classe entre étrangers et nationaux et la domination des seconds sur les premiers.

\section{Migration and (Counter)Revolution the Arab Gulf: Migration Policies and Labour Market Policies in Saudi Arabia}

In the wake of the Arab springs, the Gulf monarchies and Saudi Arabia in particular have launched counter-revolutionary policies both at the domestic and international level. Beyond the repression of political opponents, Saudi Arabia initiated a series of reforms in the field of immigration and labour market policies. Their goal was to dismiss the socio-economic discontent of Saudis and to repent a potential uprising. The political engineering involved to shape institutions and behaviours is embedded is a long term trend of politicization of migration as a key existential threat for the country. We argue that beyond regime change, the risk of a "social revolution", according to the terms of Theda Skocpol, is what looms ahead for Saudi policy makers. The reforms can therefore be understood as an effort to reinforce the class divisions between nationals and foreigners and the domination of the former on the latter.

\section{Migración y (contra)revolución en el Golfo: políticas migratorias y políticas de empleo en Arabia Saudita}

Al tiempo que las revoluciones políticas ponían en dificultad a los regímenes autoritarios de Oriente Medio, las monarquías del Golfo y, en particular, Arabia Saudita han desarrollado a escala nacional e internacional políticas "contra-revolucionarias». Más allá de la represión contra la oposición política, Arabia Saudita se lanza en una campaña de reforma de la inmigración y del empleo con la voluntad de reducir los factores estructurales que conllevan un descontento económico y social de su población y prevenir así una revolución. Auténtica ingeniería social, tales políticas se inscriben en una tendencia acentuada de politización de la inmigración desde principios de los años 1990 y de la construcción de una "amenaza migratoria" como factor de riesgo existencial contra la integridad de la nación saudita. En este artículo avanzamos la idea que, más allá del cambio de régimen, se trata en efecto de evitar el riesgo de una "revolución social» - según el término de Theda Skocpol - reafirmando las relaciones de clase entre extranjeros y nacionales y la dominación de los segundos por parte de los primeros. 